\title{
Ground and Range Operations for a Heavy-Lift Vehicle: Preliminary Thoughts
}

\author{
Luis Rabelo, Yanshen Zhu \\ University of Central Florida, 4000 Central Florida Blvd., Orlando, FL 32816
}

\author{
Jeppie Compton \\ NASA, Kennedy Space Center, FL 32899 \\ Jorge Bardina \\ NASA, Ames Research Center, CA 94035
}

\begin{abstract}
This paper discusses the ground and range operations for a Shuttle derived Heavy-Lift Vehicle being launched from the Kennedy Space Center on the Eastern range. Comparisons will be made between the Shuttle and a heavy lift configuration (SLS-ETF MPCV - April 2011) by contrasting their subsystems. The analysis will also describe a simulation configuration with the potential to be utilized for heavy lift vehicle processing/range simulation modeling and the development of decisionmaking systems utilized by the range. In addition, a simple simulation model is used to provide the required critical thinking foundations for this preliminary analysis.
\end{abstract}

\section{INTRODUCTION}

Simulation modeling is one of the most important areas for exploration. The NASA Office of Chief Technologist (OCT) [6] has stated that "Simulation focuses on the design, planning, and operational challenges of NASA's distributed, long-lived mission systems." We agree that a model represents the features of a system from a dimensional or multidimensional viewpoint. On the other hand, simulation is the execution of a model which has the possibility (if the model is able to capture appropriately the features up to certain level of fidelity) to represent its behavior. In addition, OCT states [6] that "Through the combination of the two, we can make better decisions and communicate those decisions early enough in the design and development process that changes are easy and quick, as opposed to during production when they are extremely costly and practically impossible." There are several principles with complex systems such as "emergent behavior" which can be discovered with simulation. Simulation modeling has some interesting benefits and features:

1. It helps to understand complex problems from different viewpoints: We have to understand the system and its structure, goals and objectives. We have to view complex problems from different dimensions. It is usual a multi-disciplinary effort.

2. Basic theory can be combined with experiments and expert opinions: A simulation model can fused data and information from first-principle models, empirical, and expert opinions.

3. Ontologies will be very important to increase agility and interoperability: This will support the development of knowledge discovery mechanisms and the potential automation of generating simulation models.

4. A map of models is important: A map of "models" (analytical and empirical) from the different points in the life-cycle of a system is an important endeavor in order to determine gaps.

\footnotetext{
The Engineering Meetings Board has approved this paper for publication. It has successfully completed SAE's peer review process under the supervision of the session organizer. This process requires a minimum of three (3) reviews by industry experts.

All rights reserved. No part of this publication may be reproduced, stored in a retrieval system, or transmitted, in any form or by any means, electronic, mechanical, photocopying, recording, or otherwise, without the prior written permission of SAE. ISSN 0148-7191

Positions and opinions advanced in this paper are those of the author(s) and not necessarily those of SAE. The authors are solely responsible for the content of the paper. The views expressed in this paper are solely those of the author(s) and may not reflect the views of NASA.

SAE Customer Service: Tel: $\quad 877-606-7323$ (inside USA and Canada)

Tel: $\quad$ 724-776-4970 (outside USA)

Fax: $724-776-0790$

Email: CustomerService@sae.ord
}

SAE Web Address: http://www.sae.org 
5. Find an appropriate technology platform for the simulator (e.g., distributed, cloud computing, hybrid, etc.) that makes sense and it is scalable and supports the different types of users: It is important to have configuration management and concept of operations documentation.

6. Knowledge discovery and predictive modeling are part of the simulation modeling effort: Develop different models, verification, validation and testing. Then, the discovery of relationships, according to the goals/objectives of the analysis task can be found... sometimes during the construction of the simulator many relationships are discovered.

7. Simulation has different outputs and visualization is one of them: Visualization of simulation models is very important to understand and communicate. However, scripted visualization (even it is the most advanced 3D projection) is just that "a movie". Scripted visualization is not simulation.

Indeed simulation modeling will be very important for the planning and validation of future NASA missions. This paper is just a starting point to discuss the ground and range operations for a Shuttle derived Heavy-Lift Vehicle being launched from the Kennedy Space Center on the Eastern range.

\section{CASE STUDY: ENVIRONMENT}

The NASA's Space Shuttle was the first orbital spacecraft that was a reusable launch vehicle. At launch, it consisted of the External Tank (ET), the Solid Rocket Boosters (SRB), and the orbiter (Figure 1) [2,7]. The NASA Shuttle program ended this year (2011).

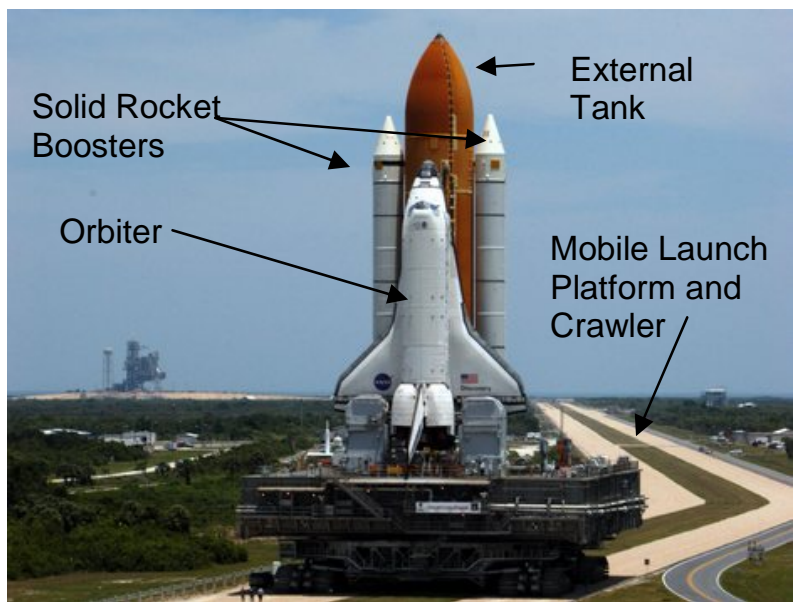

Figure 1: The NASA Space Shuttle and its main elements. Photo credit: NASA

The Shuttle launch processing was very labor intensive and consisted of three separate element flows that came together in the Vehicle Assembly Building (VAB). These three element flows required unique facilities and ground support equipment (See Figure 2).

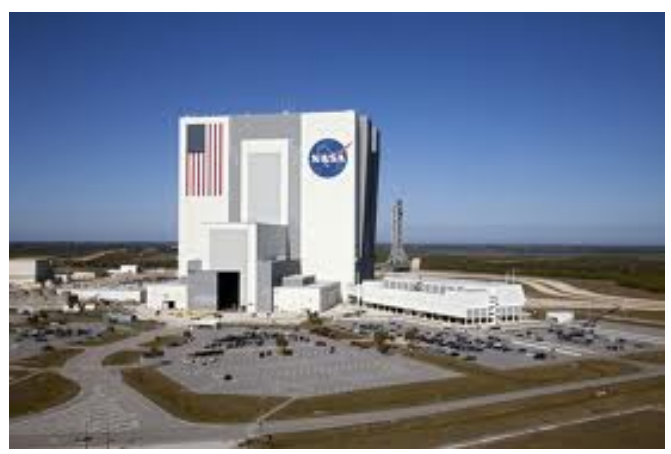

Figure 2: The Vehicle Assembly Building at KSC. Photo credit: NASA

1. The Solid Rocket Boosters (SRB) were the largest solid rockets ever designed. Each was 149 feet high and 12 feet in diameter and produced 2.65 million pounds of thrust at liftoff. Each booster consisted of four solid motor segments also called solid rocket motors, vertically stacked with a nose cone on top and the aft skirt at the base of the booster, on which the entire vehicle weight rested prior to launch

Stacking, or assembling the reusable solid rocket motors into the booster, began with transferring the aft skirt from the Rotation, Processing and Surge Facility (RPSF) to the VAB. The SRB processing flow, began when SRBs from a previous launch were retrieved from the ocean by two NASA retrieval ships and returned to Hangar AF at Cape Canaveral Air Force Station (CCAFS). Here the SRBs were disassembled and parts were returned to the vendor and other parts were refurbished for the next launch. After the SRB forward extension and aft segment were built up, they, along with the SRB segments are transferred to the VAB (See Figure).

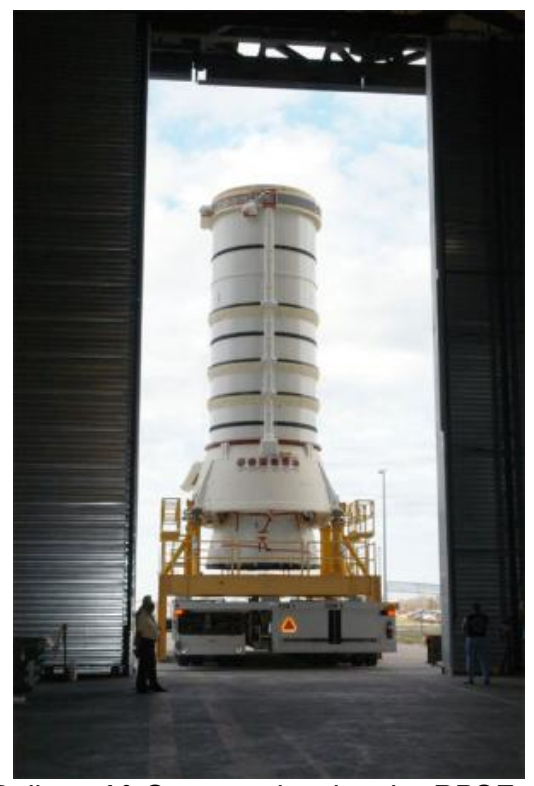

Figure 3: Built up Aft Segment leaving the RPSF on its way to the VAB, Photo credit: NASA 
2. The External Tank (ET), after arriving by barge from their assembly plant in Louisiana, were inspected and stored in either High Bay 2 or 4 until they were needed (Figure 4).

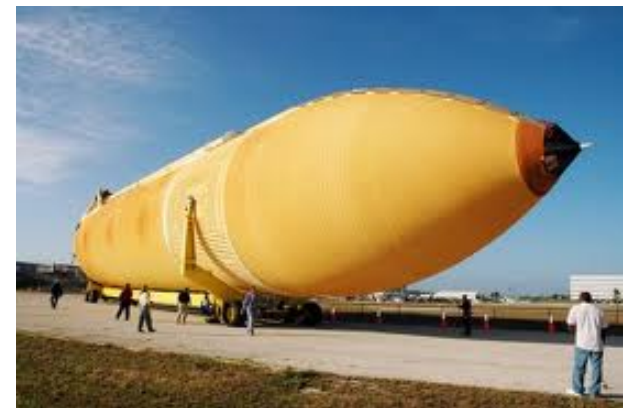

Figure 4: The External Tank rolls from the barge docked in the turn basin to the VAB. Photo credit: NASA

3. The orbiter itself was processed in the Orbiter Processing Facility (OPF). In the OPF initial access and safing was accomplished, post-flight hardware problems were resolved, thermal protection system maintenance was performed, system/component replacement and repair was conducted and Space Shuttle Main Engine (SSME) removal and installation was completed. Payload bay operations and Orbiter modifications were completed and the vehicle prepared for roll over to VAB for Vertical Operations. (Figure 5)

4.

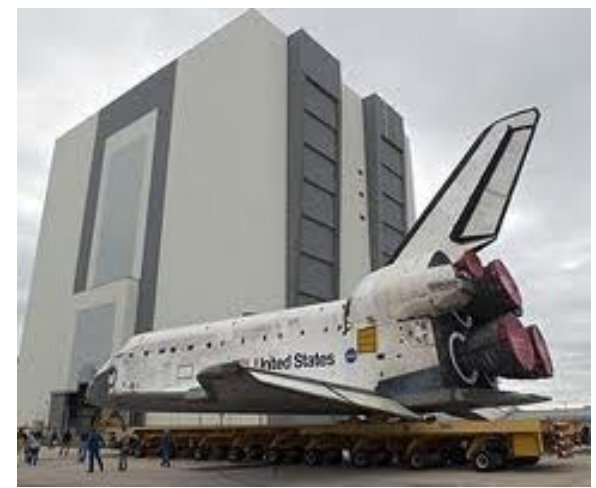

Figure 5: Shuttle Orbiter rolling over from the OPF to the VAB. Photo credit: NASA

Once a Mobile Launch Platform (MLP), a two-story tall, nine-million-pound steel structure that serves as the launch base for the integrated Shuttle stack was in place in the high bay, SRB stacking began and required approximately three weeks. This was accomplished by hoisting the Solid Rocket Motors onto the MLP in either High Bay I or 3 and the segments individually mated to form two complete SRBs.Once the SRB stacking was complete, ET mate and closeout, which requires approximately two weeks, was completed (Figure 6)

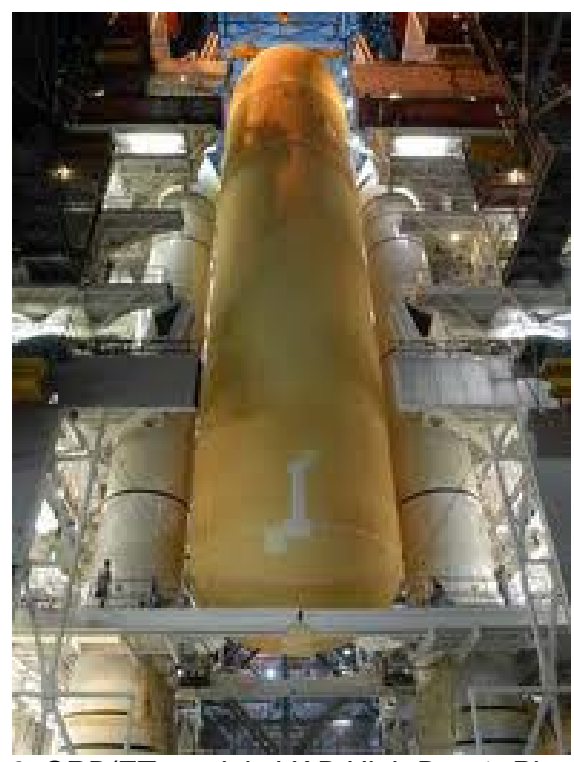

Figure 6: SRB/ET stack in VAB High Bay 1. Photo credit: $N A S A$

At this time, the orbiter was rolled over from the OPF and parked in the transfer isle (Figure 5). A strong-back was attached to the orbiter and it is lifted to near the top of the $V A B$, moved into the high bay and lowered into position for mating with the ET/SRB stack. Mating the orbiter to the ET/SRB stack required approximately one week. The integrated stack was now complete and interfaces between the three Shuttle elements were tested and structural closeout was performed. The integrated stack was now ready to roll out to the launch pad.

Pad processing at Launch Complex (LC) 39A or 39B takes approximately 4 weeks. Here the payload is transferred from the payload canister to the Payload Change-out Room (PCR) and then to the Orbiter. The Shuttle/Pad system is validated and simulated launch count down run with participation of the astronauts. Finally preparations are made for the vehicle to respond to launch countdown (Figure 7).

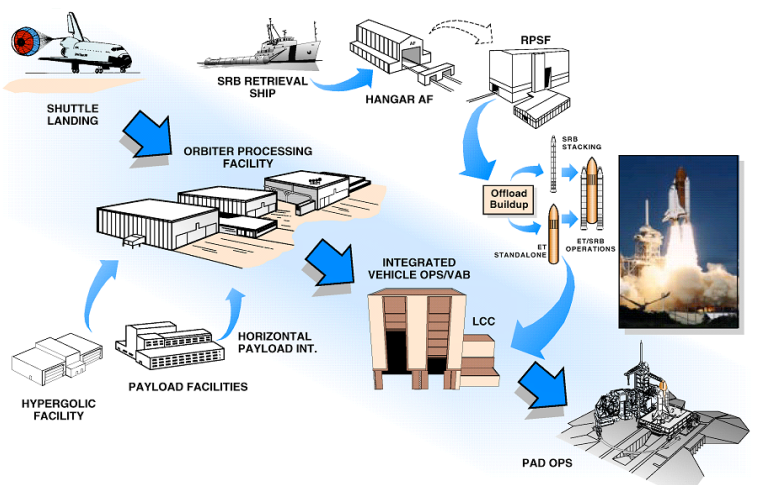

Figure 7: The standard flows to process the three elements of the Space Shuttle. Source: NASA

POTENTIAL VEHICLES - NASA has announced that the next manned spacecraft will be the Multi-Purpose Crew Vehicle (MPCV), which is based on the Orion, the Apollo era crew capsule design selected by the now- 
canceled Constellation program. The MPCV will provide 316 cubic feet of habitable space, carry four astronauts on missions of up to 21 days that extend beyond lowEarth orbit and will land off the California coast in the Pacific Ocean.

The MPCV and a Heavy Lift Vehicle (HLV) (Figures 8 and 9) are central to NASA's plan for the future of space exploration beyond LEO. NASA's goal is to develop exploration architecture consistent with direction in the NASA Authorization Act of 2010. The Act gives NASA until 2016 to field the heavy-lift rocket and crew vehicle and authorizes approximately $\$ 10$ billion in spending on the two projects over the next three years.

To meet the above goal, NASA plan to implement the MPCV and SLS Programs, including transition of relevant design and developmental activities of the Constellation Program. A major element of the transition involves shifting design and developmental efforts away from a closely coupled system (Ares I and Orion) to a more general launch vehicle (i.e., SLS) and crew vehicle (i.e., MPCV, Figure 8 and 10).

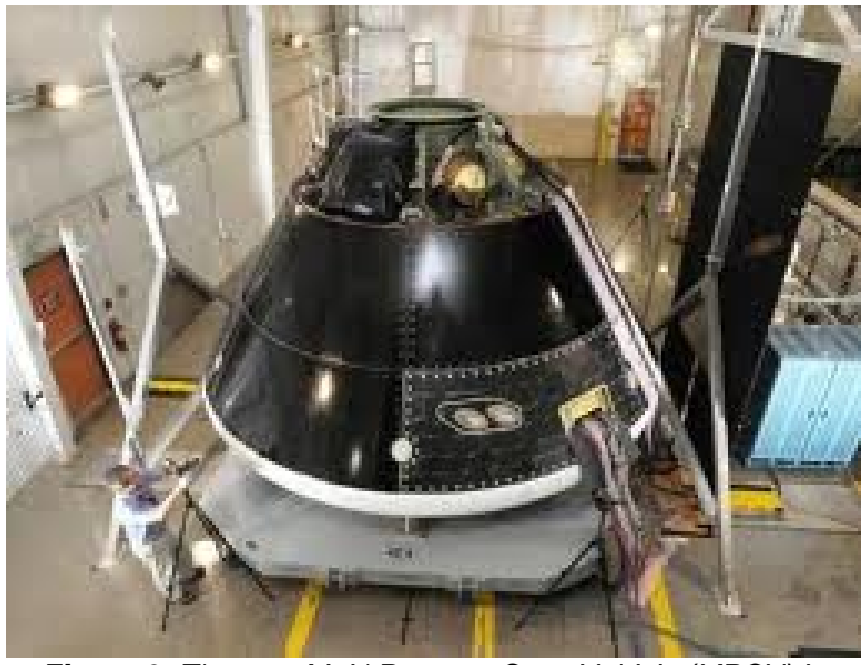

Figure 8: The new Multi Purpose Crew Vehicle (MPCV) in production. Photo credit: NASA

To be successful both as an individual program and as a component of an affordable exploration architecture, the SLS program must greatly reduce development and operations costs from NASA's experience in past programs. Affordability, and crew and public safety, are primary objectives for heavy-lift, NASA, with support from industry partners, will investigate alternative vehicle designs and architectures to validate, support, or challenge design plans, ensuring an affordable design that meets NASA's requirements.

In FY 2012, NASA will continue to define a sufficiently affordable, sustainable and realistic SLS development plan. NASA will leverage existing designs and hardware which includes NASA's selection of an SLS Reference Vehicle Design (RVD) that is derived from Ares and Shuttle hardware. Informed by the NASA analyses, the current RVD is a 27.5-foot diameter core liquid oxygen/liquid hydrogen (LOX/LH2) vehicle with five Space Shuttle Main Engine (SSME)-derived core stage engines, (designated RS-25E), a LOX/LH2 upper stage with a J-2X engine, and two Ares-derived five-segment solid rocket boosters. The RVD would provide a combined lift capability of approximately 100-130 metric tons to LEO (Figure 9).

Shuttle ET diameter
4 or 5 segment PBAN booster, evolvable to HTPB
SSME (RS-25D) transitioning to
RS-25E

Figure 9: One of NASA's SLS reference vehicle design HLV configurations. Source: NASA Architecture 5.0 Briefing

The advantage of this HLV configuration is that it can utilize much of NASA's existing Space Shuttle launch and processing infrastructure (with modifications) launch pads, mobile launcher, scaffolding in the VAB and fabrication facilities in Michoud, MS and at the vendor in Utah. However, the entire infrastructure is not required due to several changes to the launch scenario. For example, the SRBs are not planned to be recovered so neither the SRB retrieval ships nor Hangar AF are needed.

The initial capability of the core elements, without an upper stage, should be for between 70 tons and 100 tons into LEO in preparation for transit missions beyond lowEarth orbit. With the addition of an integrated upper Earth departure stage the total lift capability of the SLS should be 130 tons or more. It is expected to be operational by 2016 .

For the purposes of this paper we are assuming the SLS HLV will be processed similar to the way the Shuttle was processed: The SRMs will still arrive by train, be processed through the RPSF and ARF then transferred to the VAB for stacking on the Mobile Launcher. The vehicle core and upper stage will arrive by barge or possibly by air. They too will be transferred to the VAB for integration with the SRB stack. Final Assembly of the MPCV will take place in KSC's Operations \& Checkout Building $(\mathrm{O} \& \mathrm{C})$ and be transferred to the VAB for integration atop the HLV stack. Once integration, test and checkout are complete, the stack will be taken to Pad 39 A or B via a Shuttle era crawler for final launch operations. 


\section{CASE STUDY: SIMULATION CONFIGURATION}

The operational simulation and its details will be accomplished by using different paradigms: agents, discrete-event, continuous, and system dynamics. However, the object-oriented framework will be the basis for this. The paradigm of agents will be the driver of the ground processing and range simulator. The interactions of the decision-makers, the life cycle of the mission, the features (dynamics, reliability, behavior) of the most important resources, and other systems (e.g., from nature (weather) drive this simulation. Therefore, in this paper we will focus on the agent-based configuration.

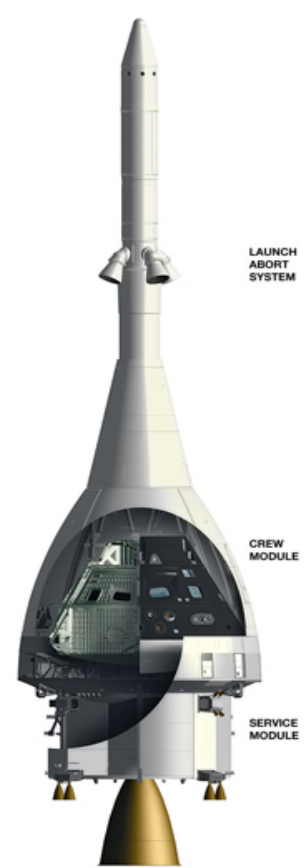

Figure 10: Four-person Multi-Purpose Crew Vehicle (MPCV) with an escape rocket and a service module (http://www.cbsnews.com/network/news/space/exploration/gra phics/mpcv/mpcv stack vert.jpg).

SIMULATION WITH AGENTS - Agent-based simulations are implemented by using an object-oriented framework, allowing for detailed modeling of the different elements. The simulation of a NASA mission using SLSETF with MPCV will combine several agents, define their relationships, and observe their resulting interactions over time. There are different types of agents: Resource/System-Agents, Process Agents, and Decision-Maker agents.

Decision-Maker Agents -This type of agents make decisions. These agents include the common representations of discrete-events such as queues and clocks, finite state machines, differential equations, and others. They also include constructs to make decisions such as expert systems, neural networks, and other mechanisms from operations research. We have to investigate the most important actors in the ground and range processes and their interactions (e.g., messages, reporting, hierarchies, and collaborations) with other agents. Actors such as the Range Safety Officer, Weather Officer, Entry Flight Director, Launch Director, Spacecraft Commander, Test Director, Payload Manager, Launch Integration Manager, Lead Flight Director, and Chairman of the Mission Management Team are very important to be modeled. Examples of these actors are explained below:

Range Safety and Weather Officers - Agents: The range safety and weather officer agents are examples of decision-maker agents. A range safety officer function is to provide the Expectation of casualties $\left(E_{c}\right.$ - collective risk - it is mainly a safety metric) and the weather officer provides the weather impact $[5,8]$. Both are necessary for making a decision about the launch. Range operations is an essential element of space operations. The range is considered to be the volume through which the space vehicle must pass on its way to and from space, and its projection on earth. The range encompasses many different operations (security, weather, facilities, vehicle processing, and safety) (Figure 11); like many of the operations, continuous routines simulate range safety to obtain answers from blast, toxic, debris, and flight trajectory models. Weather also has a high level of complexity. Here the general process can be represented by discrete-event steps which will have to call routines to gather timely weather observations and forecast (short and long term). In addition, several databases will have to be accessed which represent the geographic and demographic information (geography, traffic, population behavior, and shelter references), and the reliability features of the different components of the SLS-ETF and the MPCV. The clock of the discrete-event process will drive the "calls" to the different components and their important coordination. In addition, this agent will have to provide the data and information fusion in order to provide respective results $[4,8]$. An expert system can also be used in order to make Go/No Go decisions based on $E_{c}$, the characteristics of clouds, and weather patterns.

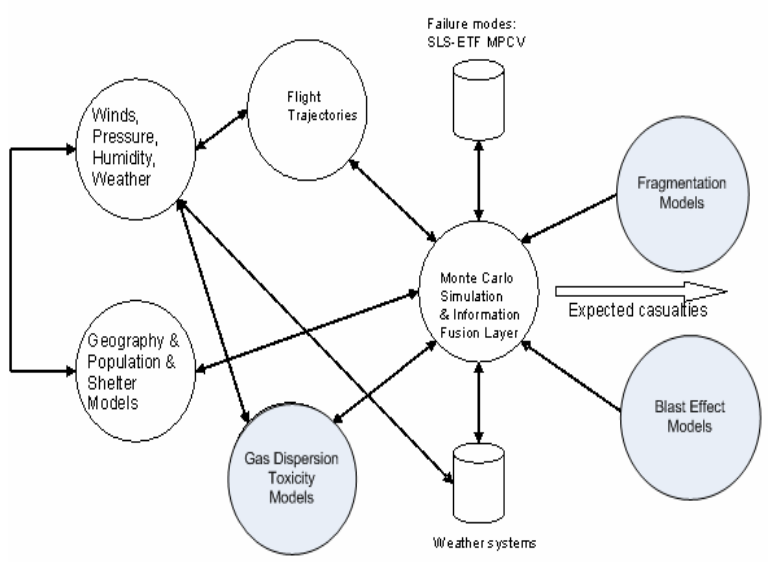

Figure 11: Interactions of different information systems to be consulted by range safety and weather officer Agents.

Launch Director - Agent: The Launch Director agent is the one that gathers information about subsystems such as Orbital Maneuvering System, the Reaction Control 
System (RCS); Electrical Power; Hydraulic Power; Environment Control and Life Support System, Thermal Protection, Propulsion (main engines, solid boosters), etc. The Launch Director agent based on the health monitoring and testing of these systems can provide Go/No Go decisions and establish orders to repair the systems, and a new baseline for new dates to be launched consulting with the Lead Flight Director Agent, the Range Safety and Weather Officer Agents, and the Launch Integration Manager Agent.

Resource/System Agents - These agents utilize differential equations, continuous models, and discreteevent flows in order to simulate their behavior, aging, and availability/serviceability. They represent important systems of the SLS-ETF (e.g., solid rocket boosters, main engines, etc.) and the MPCV (e.g., service module, ablative thermal protection system, etc.). In addition, they can represent important resources (e.g., Vertical Assembly Building (VAB), launch pad, etc.) and other spacecrafts (e.g., International Space Station (ISS)).

$V A B$ - Resource Agent: The VAB is a resource agent. It is the place where the integration (stacking) process occurs. The VAB is not only a building with dimensions but also it has a workforce and other resources such as overhead bridge cranes and lifting devices.

International Space Station (ISS) - Resource Agent: The ISS is an agent resource. The ISS provides a location in Low Earth Orbit (LEO) to test spacecraft systems required for long-duration missions to asteroids (Near Earth Asteroid (NEA) exploration) and Mars [1]. ISS provides a place with capabilities for maintenance, repair, and replacement of systems on-orbit (Figure 12). ISS will be essential in operating spacecraft such as the MPCV further from Earth. One of the missions for SLSETF MPCV will be to commute from earth to the ISS in order to rotate crews and provide basic services and supplies to the ISS. Docking and undocking by MPCV will be performed at the ISS.

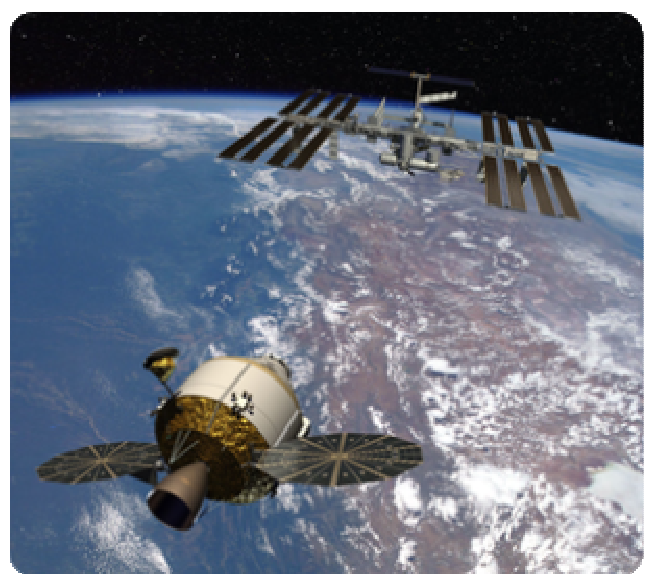

Figure 12: Rendezvous of MPCV-Service Module with ISS (http://www. americaspace.org/wpcontent/uploads/2010/04/OrionRendezvous/SS.png).
Solid Rocket Booster - Resource Agent: The two SRBs (five segments) will provide important thrust to lift the SLS-ETF off the launch pad (Figure 13). The SRBs are large solid-propellant motors. Primary elements of each booster are the motor (including case, propellant, igniter and nozzle), structure, separation systems, operational flight instrumentation, pyrotechnics, thrust vector control system and range safety destruct system. The propellant is commonly referred to as Ammonium Perchlorate Composite Propellant, or simply APCP.

Process Agents - These agents utilize mainly discreteevent flows such as discrete-event simulation using discrete-event lists and/or state charts. They are very important to represent the different phases of the life cycle of a mission and/or the different steps of a complex process. They can have environments. Other agents can be an active part of the phases and collaborate with other agents using that specific phase/environment. This type of agent is useful to represent a mission or a process such as the mating (stacking) process.

Mating Process - Process Agent: This is basically an assembly discrete-event process. However, the advantage of using the agent framework is the assignation of environments and features which allow other agents to use the environment to participate and collaborate with other agents in the process. The following steps are required:

1. Phases 1 and 2 Transfer to VAB: The first phase and second phases arrives at KSC. They are inspected, then off-loaded and towed to the VAB transfer isle where they are stored until integrated with the SRB stack.

2. SRB Stacking in the VAB High Bay: The Solid Rocket Motor (SRB) stacking consists of placing an SRB's aft skirt onto hold-down posts on the Mobile Launch (MP) in one of the VAB High Bays (HB). The SRBs are then stacked one segment at a time until all five segments are stacked. At this time the forward extension is added that houses the avionics and parachutes and SRB stacking is complete.

3. Phases 1 and 2 are assembled and mated to the SRB stack in the VAB: This is accomplished by raising the phases to a vertical position in the transfer isle, lifting it up and over into the HB and Mating it to the stacked SRBs.

4. MPCV with Service Module to VAB: The MPCV is towed to the $V A B$ and placed in the VAB transfer isle. A strong-back is attached to the MPCV and service module and the vehicle is lifted up and moved, lowered and attached (mated to Phase 2) to the Phase 1/Phase 2/SRB stack.

Mission - Process Agent: The Mission Process Agent is the heart of the simulation. It describes the life cycle of a mission and owns different environments where the different decision-maker agents, resource agents, and 
other process agents can work together and collaborate. It is an assembly discrete-event process. However, the advantage of using the agent framework is the assignation of environments and features which allow other agents to use the environment and participate and collaborate with other agents in the process. The following steps are required (Figure 14):

1. Supply Chains, Rollover and VAB: This step in the life cycle of the Mission details the different resources and systems to be delivered to the VAB for mating (Stacking). It might involve details of the internal supply chain in NASA (i.e., the interactions between NASA Centers and HQ for the Mission) and the external supply chain (i.e., the interactions between NASA and Major Contractors). In addition, the rollover of the major systems and the different processes to occur inside the VAB. One of the environments is the VAB environment.

2. Rollout: This step is very short in time. The vehicle is transferred from the VAB to the launch pad.

3. Launch Operations: This step includes pre-launch operations to be performed on the vehicle already on the launch pad. There are many interactions among different agents. The decision-maker agents such as the launch director, range safety and weather officers, the crew technician agents, are heavily involved during this step. Scrubs are simulated and the assignment of potential launch dates is also modeled. The weather and the range systems are executed accordingly. The final launch is modeled.

4. Ascent Phase: This is a step with a short period of time. It simulates the SRBs and the phases being released.

5. Orbiting, Rendezvous, Docking, Orbit Operations, and Undocking: This step simulates the orbit, rendezvous, and docking of the vehicle (MPCV and the service module) with the ISS. Undocking and the planning of the reentry and landing (interactions of the different agent decision makers such as the entry flight director, weather and range safety officers, etc.) are simulated.

6. Orbiting, Entry, and Landing/Recovery: This is the final step of the Mission with the final orbiting, the release of the service module, and the entry and landing at a particular location (e.g., California Coast) and the logistics of the recovery.

\section{CASE STUDY: PRELIMINARY PROTOTYPE}

A preliminary prototype of a system with just a limited numbers of agents was developed. The system implements the Mission (Figure 14) as the driven source of the entire simulation. The Delivery_Rollover_VAB "state" is the one simulated with more detail in the prototype. This "state" is very discrete-event in nature due to the assembly operations.

The simulation platform selected is AnyLogic (www.xjtek.com). An "Agent" in AnyLogic is a unit of model design that can have behavior, memory (history), timing, and contacts. Agents can represent people, companies, projects, assets, vehicles, cities, animals, ships, products. AnyLogic has classes for developing agents as it has all necessary properties to define variables, events, statecharts, System Dynamics stock and flow diagrams.

Design of an agent typically starts with identifying its main drivers and interface with the external world. The agents decision-makers can use ports as agent interface points. In case of large number of agents with dynamic connections (such as organizational hierarch, e.g., Mission Management Team) agents can communicate by calling methods of each other or through the environment.

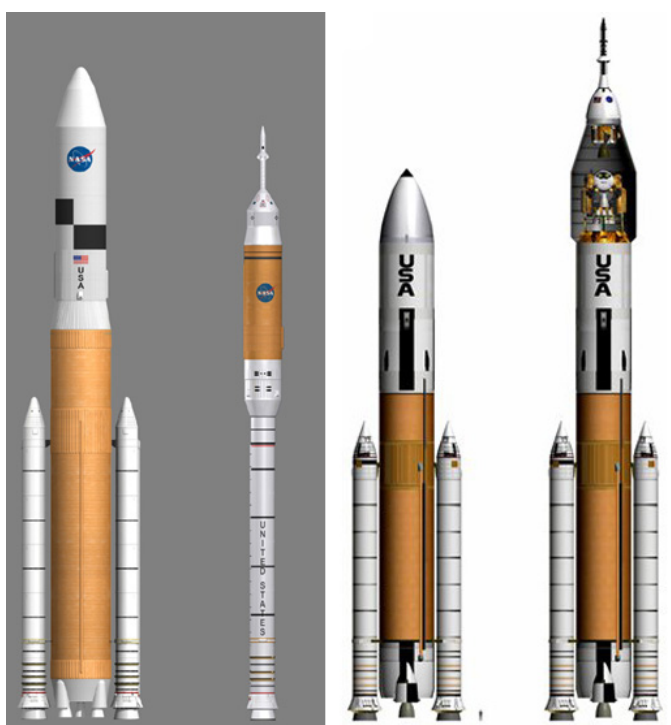

Figure 13: The SLS is an integrated evolution of Apollo, NASA Shuttle, and the Constellation Programs (http://dvice.com/pics/nasa showdown.jpg).

This model can be deployed using any hardware/software system. 


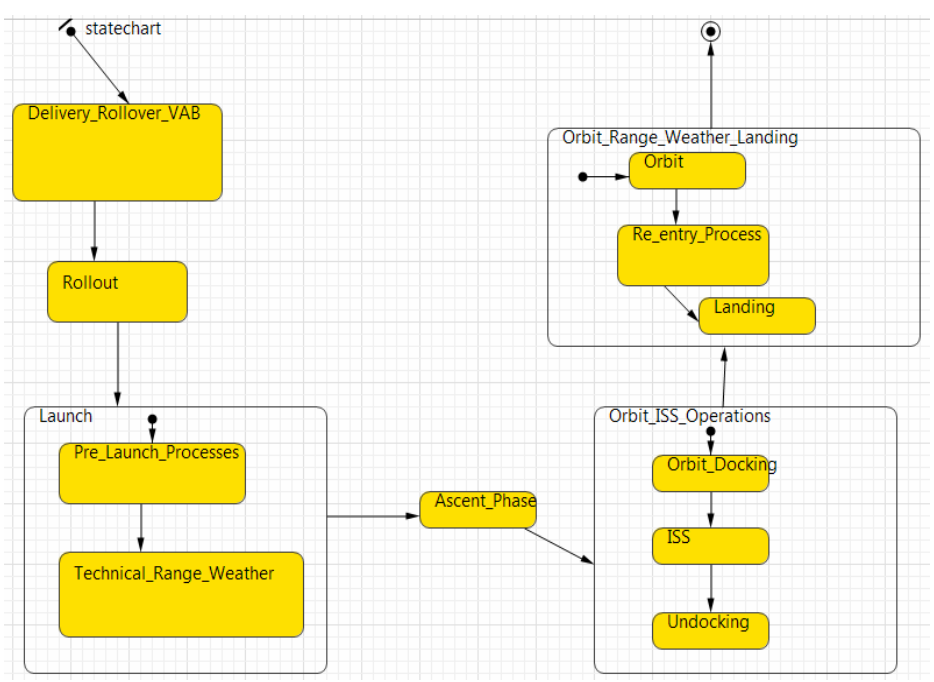

Figure 14: State chart of a Mission (LEO) using AnyLogic.

\section{CONCLUSIONS AND FURTHER WORK}

A simulation modeling configuration driven by agents and using the four paradigms is the right start for a comprehensive simulation tool for the SLS. In addition, components of the digital twin are able to be used and the planning for other type of missions (e.g., NEA). This approach can be used for planning at different levels (strategic, operational, and tactical). It is very important to appreciate the level of integration to be achieved with other information systems and the real-time issues involved in particular for advanced digital twin concepts. Scripted visualization is important but it does not compare along with visualization of the simulation. This paper outlined some of the preliminary thoughts that will evolve when more details are available.

Simulation modeling can develop baselines and trade studies for the different systems being developed and proposed (Figure 15). It is well known that when NASA stopped flying the space shuttle, the US no longer has a vehicle to carry humans to space. Therefore, the commercial industry is trying to fill the gap with different types of solutions/services.

\section{ACKNOWLEDGMENTS}

We would like to give thanks to the Kennedy Space Center (KSC) for the different learning experiences provided. The views expressed in this paper are solely those of the author(s) and may not reflect the views of NASA.

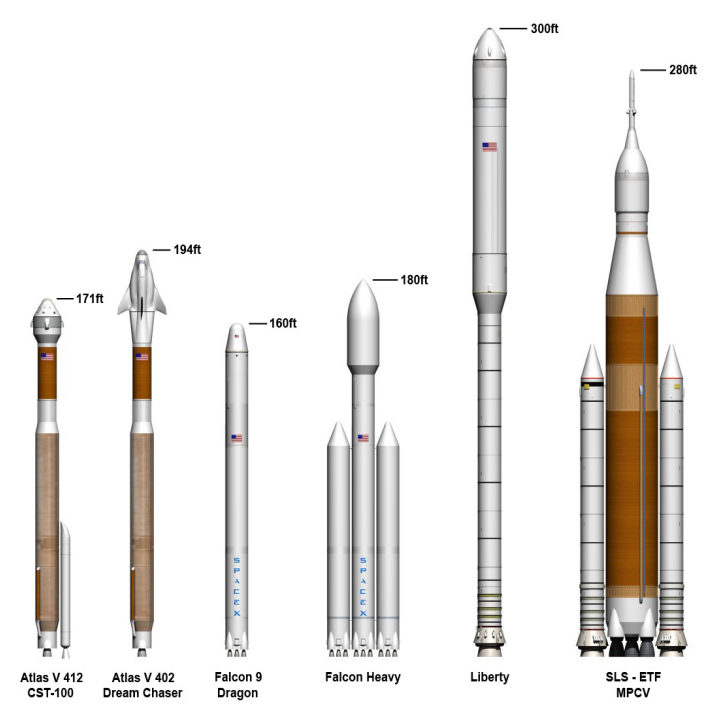

Figure 15: Spacecrafts from different companies and the STSETF MPCV from NASA.

\section{REFERENCES}

1. Adamo, D., Giorgini,J., Abell, P. and R. Landis (2010), "Asteroid Destinations Accessible for Human Exploration: A Preliminary Survey in Mid-2009," Journal of Spacecraft and Rockets, Volume 47, 9941002.

2. CAIB Report (2003), Columbia Accident Investigation Board, Determination of Debris Risk to the Public Due to the Columbia Breakup during Reentry.

3. Haler, D., (1987) "Statecharts: A visual formalism for complex systems", Science of Computer Programming, Volume 8, 231-274.

4. Hall, D. and J. Llinas, (1997), "An introduction to multisensor data fusion," Proceedings of the IEEE 85[1], 6-23.

5. Rabelo, L., Sepulveda, J., Compton, J. and R. Turner (2006) "Simulation of Range Safety for the NASA Space Shuttle," Aircraft Engineering and Space Technology Journal, Volume 78[2], 2006, 98106.

6. NASA Office of the Chief Technologist (OCT) (2010), DRAFT Modeling, Simulation, Information Technology \& Processing Roadmap Technology Area 11 http://www.nasa.gov/pdf/501321main TA11MSITP-DRAFT-Nov2010-A1.pdf.

7. Review of U.S. Human Spaceflight Plans Committee (2009) Seeking a Human Spaceflight Program Worthy of a Great Nation, NASA Publications.

8. Sala-Diakanda, S., Sepulveda, J. and L. Rabelo (2009) "An information fusion-based metric for space launch range safety," Information Fusion Journal, (accepted for publication). 


\section{CONTACTS}

LUIS RABELO, Ph.D., is an Associate Professor in the Department of Industrial Engineering and Management Systems at the University of Central Florida. He received dual degrees in Electrical and Mechanical Engineering from the Technological University of Panama and Master's degrees from the Florida Institute of Technology and the University of Missouri-Rolla. He received a Ph.D. in Engineering Management from the University of Missouri-Rolla, where he also did Post-Doctoral work in Nuclear Engineering in 1990-1991. He holds dual MS degrees in Systems Engineering \& Management from the Massachusetts Institute of Technology.

YANSHEN ZHU is a Ph.D. student at the School of Electrical Engineering and Computer Science of University of Central Florida where he received his M.S. degree in Computer Engineering in 2003.

JEPPIE COMPTON is a NASA Senior Engineer at the Kennedy Space Center. He is a retired Air Force officer with a bachelor's degree in Meteorology from the University of Utah and a master's degree, also in Meteorology, from St Louis University. He has considerable experience in Program Management on the Space Shuttle program, numerous satellite programs and with Unmanned Vehicles (air and sea) programs. He managed Houston Systems Engineering, McDonnell Douglas Space Station work package II and the Human Life Sciences Projects Office for Lockheed Martin at the Johnson Space Center. He has a long history in modeling and simulation having hosted the first Meteorological Interactive Processing Conference in Boulder, CO in 1981.

JORGE BARDINA, Ph.D., is a research scientist in the Intelligent Systems Division at NASA Ames Research Center. His areas of interests are real-time collaborative environments, web-based and parallel architectures, intelligent systems, modeling, simulation, and engineering technologies. Author of over 80 publications in the areas of intelligent systems, information sciences, aeronautics, aerothermodynamics, computational fluid dynamics and turbulence. Dr. Bardina received his Civil Engineering of Industries with Mechanical Mention degree from Universidad Catolica de Chile and his Ph.D. degree and M.S. degree on Mechanical Engineering and M.S. degree in Economics from Stanford University. 